\title{
A Modular Toolkit to Fabricate Microfluidic Devices for 3D Cell Culture and Near Real- time Measurements
}

Giraso Keza Monia Kabandana ${ }^{1}$, Adam Michael Ratajczak ${ }^{1}$, and Chengpeng Chen ${ }^{1 *}$

1. The department of chemistry and biochemistry, University of Maryland Baltimore County

${ }^{*}$ Corresponding to

Dr. Chengpeng Chen

Department of Chemistry and Biochemistry

University of Maryland Baltimore County

Baltimore, MD, 21250

cpchen@umbc.edu 410-455-3053 


\begin{abstract}
Microfluidic technology has tremendously facilitated the development of in vitro cell cultures and studies. Conventionally, microfluidic devices are fabricated with extensive facilities by well-trained researchers, which hinders the widespread adoption of the technology for broader applications. Enlightened by the fact that low-cost microbore tubing is a natural microfluidic channel, we developed a series of adaptors in a toolkit that can twine, connect, organize, and configure the tubing to produce functional microfluidic units. Three subsets of the toolkit were thoroughly developed: the tubing and scoring tools, the flow adaptors, and the 3D cell culture suite. To demonstrate the usefulness and versatility of the toolkit, we assembled a microfluidic device and successfully applied it for 3D macrophage cultures, flow-based stimulation, and automated near real-time quantitation with new knowledge generated. Overall, we present a new technology that allows simple, fast, and robust assembly of customizable and scalable microfluidic devices with minimal facilities, which is broadly applicable to research that needs or could be enhanced by microfluidics.
\end{abstract}




\section{INTRODUCTION}

Microfluidics are miniaturized devices that manipulate small amounts of liquid within flow channels on the 1-1000 $\mu \mathrm{m}$ scale ${ }^{1}$. This technology has tremendously facilitated developments in chemical measurement ${ }^{2}$, separation ${ }^{3}$, and synthesis ${ }^{4}$ in the past decades. In particular, microfluidics provide a dynamic platform for cell cultures ${ }^{5}$. Compared to static cell cultures in flasks/dishes, the dynamic flow enables continuous oxygen/nutrient supply and waste removal, thereby maintaining a fresh microenvironment for cells therein ${ }^{6}$. More importantly, the flow can introduce shear stress, which is critical for certain cell types, such as endothelial cells ${ }^{7}$. This technology is often referred to as organs-on-a-chip, and it holds the potential to revolutionize disease modeling and drug testing by mimicking the behaviors of tissues/organs in vivo $6,8,9$.

A milestone in the fabrication of microfluidic devices is the invention of soft lithography by the Whitesides group ${ }^{1}$. Briefly, in a clean room, a master replica mold (typically on a silicon wafer) with raised microstructures is prepared by using photomasks, photoresist resin, and photoirradiation; the prepolymer of PDMS (polydimethylsiloxane) is poured onto the mask followed by heat-stimulated curing to replicate the microstructures; the PDMS slab can then be sealed onto another piece of PDMS or glass, thereby forming enclosed microfluidic channels ${ }^{10}$. Recently, 3D-printing has been exploited to fabricate microfluidic devices with complex microstructures ${ }^{11}$. Compared to PDMS-based microfluidics, 3D-printed devices are more robust, standardized (e.g. threaded connecting ports), and customizable ${ }^{12}$. However, there are limitations including the relatively low resolution of current 3D-printers and the resulting rough surfaces of the microchannels ${ }^{13}$.

Currently, microfluidic technology is predominantly found in highly speciallized laboratories in academia-wider translation is hindered by the high facility, time, and personnel requirements needed for fabrication of the devices ${ }^{14}$. The fact that microfluidic devices are becoming more and more integrative worsens the situation. Fully integrated devices are inflexible for post-fabrication modification, and if any part fails, the whole device must be discarded, which further increases the costs. The concept of modular microfluidics, which allows building blocks to be assembled by users for the production of a desired microfluidic device, has been proposed as a possible solution to these problems ${ }^{14-16}$. However, while it is true that modular microfluidics are more flexible for customization and adjustments, current technologies do not offer opportunities for widespread adoption of the microfluidic devices. High-end facilities are still required to fabricate well-defined microchannels in the modules, and these are usually single-use blocks due to the 
arduous process of cleaning the microchannels therein. Moreover, a universal protocol outlining the development of an easy connection between the modules for precisely delivering liquids on the $\mu \mathrm{L}$ scale has not been formulated. Therefore, in this work, we present our invention of a modular toolkit for easy assembly of functional microfluidic devices without fabricating microchannels. Instead, disposable microbore tubing was used as the microchannel, and microadapters were 3D-printed to connect and/or adapt the tubing to form microfluidic units. Utilizing the flexibility of the tubing, the well-characterized ports in the adapters allowed for plugand-play and leakage-free connections. The adapters were reusable and the low-cost tubing (microchannels) could be discarded and replaced. We demonstrated the versatility of this technology by assembling a microfluidic device using the toolkit, which integrated 3D cell culture, fluids joining/mixing, and near real-time quantitation.

\section{EXPERIMENTAL}

\section{Design and fabrication of the microadapters}

All microadapters were designed in Autodesk Inventor 2019 (CA, US). The detailed engineering sketches are included in the supplementary information (SI; Figs. S2-S3). The design files were uploaded to our MJP 5600 3D-printer (3D Systems, SC) for fabrication. The VisiJet CRCL200 material was used, the composition of which is proprietary but is known to be acrylatebased.

\section{Connecting microbore tubing to the adapters}

Microbore Tygon tubing (inner diameter $=0.5 \mathrm{~mm}$; outer diameter $=1.5 \mathrm{~mm}$; Cole-Parmer, $\mathrm{NJ}$ ) was used as disposable microchannels. The ends of the tubing were scored flat to avoid dead volumes after assembly. As shown in Fig. 1A, a scoring guider was used for this purpose. Fig. $\mathbf{S 1}$ in the SI demonstrates the process. A piece of tubing was placed through a hole $(1.6 \mathrm{~mm}$ diameter, slightly larger than the o.d. of the tubing) in the guider, followed by being scored using a sharp blade along the surface of the guider.

Two types of flow adapters were used: tubing configurators and connectors. Tubing configurators had holes or circular grooves for holding the tubing in certain configurations. Connectors had ports where the tubing could be connected. For the configurators, the diameter of the holes and the grooves was $1.5 \mathrm{~mm}$, the same as the o.d. of the tubing. For connectors, 
connection ports of $1.4 \mathrm{~mm}$ were used so that the tubing could be crumpled in tightly without leakage.

\section{Measuring the mixing efficiency of the serpentine adapter}

Red and green food color solutions (1\% in PBS) were delivered into the tubing serpentine on the adapter by two syringe pumps and a T-mixer at $6 \mu \mathrm{L} / \mathrm{min}$. Microscopic pictures of the solutions in the tubing were taken and analyzed in the software ImageJ. The area of the red stream was used as the measurement of mixing efficiency. At the beginning of the mixing (1st path of the serpentine), the red stream took $50 \%$ of the tubing width, which was normalized to $0 \%$ mixing. If no red was visible, or the area of the red stream was 0 , a $100 \%$ mixing was achieved. By this scale, the mixing efficiency was quantitated.

\section{The 3D cell culture modules}

The suite included a holder, a lid, and a fibrous insert. The holder was 3D-printed from a flexible material (CE-NT; composition is proprietary; 3D Systems, SC) with a concave obround slot of $15 \mathrm{~mm}$ long, $5 \mathrm{~mm}$ wide, and $3.5 \mathrm{~mm}$ deep. A $3.0 \mathrm{~mm}$ high matching lid was fabricated from the rigid material with two holes $(1.4 \mathrm{~mm}$ diameter) to connect tubing. The fibrous insert was prepared by electrospinning a layer $(50 \mu \mathrm{m}$ thick) of polycaprolactone (PCL) fibers on a polystyrene film (200 $\mu \mathrm{m}$ thick) following the previously reported protocoli7. Upon using, the cellladen insert was placed in the holder with the fiber side facing up. After the lid was inserted into the slot of the holder, a $250 \mu \mathrm{m}$ space was formed between the insert and the end of the lid, thereby allowing for flow through the channel.

\section{Computational fluid dynamics (CFD) simulation}

CFD simulation was conducted in the software of Autodesk CFD (Autodesk, CA). Acrylate was assigned as the material of the cell chamber and PBS was used to simulate the media. The boundary and initial conditions were defined by flow rate, which was $6 \mu \mathrm{L} / \mathrm{min}$.

\section{Assembling the microfluidic device and characterizing its analytical merits}

Fig. 5A shows the assembled microfluidic device. To characterize the analytical merits of the device, nitrite standards of $0.00,1.25,2.50,5.00$, and $10.00 \mu \mathrm{M}$ prepared in phenol red-free $\mathrm{L}-15$ media were pumped through the cell chamber (with the insert but no cells) at $6 \mu \mathrm{L} / \mathrm{min}$ towards the T-mixer, where the Griess reagent was mixed in by another syringe pump at the same flow rate. The spectrometer connected to the detector tubing via optical fibers was set to take 
absorbance measurements every $20 \mathrm{sec}$ at $520 \mathrm{~nm}$ and $600 \mathrm{~nm}$. The $520 \mathrm{~nm}$ was the maximum absorbance wavelength while the $600 \mathrm{~nm}$ was used to calibrate any baseline drifting by e.g. the light source. The (Abs520-Abs600) was used as the signal. Each standard was flowing for about $10 \mathrm{~min}$ before the next concentration was loaded. The average signals of the standards were plotted as a function of the concentrations. The $\mathrm{R}^{2}$ was calculated by the least square regression method. The detection limit (LOD) was calculated by multiplying 3 with the standard deviation of the blank and dividing the result by the slope of the calibration curve.

\section{Macrophage culture and measurements}

Ten inserts were placed in a $60 \mathrm{~mm}$ petri dish with the fiber side facing up, which was then filled with $5 \mathrm{~mL} 70 \%$ ethanol. The inserts in the petri dish were placed in a UV biohood overnight for the ethanol to dry. The commonly used RAW264.7 macrophage cell line was used for this study $^{18}$. Cells cultured in a T75 flask between passages 3 and 5 were removed by a scraper and resuspended in L-15 media to a final density of $2 \times 10^{6}$ cells per $\mathrm{mL}$. An aliquot of $2 \mathrm{~mL}$ of the cell suspension was pipetted onto the sterilized inserts. The dish was then incubated at $37^{\circ} \mathrm{C}$ (humid; $5 \% \mathrm{CO}_{2}$ ) for 6 hours, after which, $5 \mathrm{~mL}$ fresh media was added. The cells were cultured for another 48 hours with daily media change.

When a cell-laden insert was placed in the chamber, phenol-red free media with or without lipopolysaccharide (LPS, $1 \mu \mathrm{g} / \mathrm{mL}$; Millipore-Sigma, MO) was pumped through at $6 \mu \mathrm{L} / \mathrm{min}$ for three and a half hours. The cell chamber was placed in a desktop humid incubator at $37^{\circ} \mathrm{C}$. Any nitrite from the macrophages was brought out by the flow to the T-mixer to mix with the Griess reagent $(6 \mu \mathrm{L} / \mathrm{min})$. After further mixing in the serpentine, absorbance was measured using the same protocol as described for the nitrite standards. After each run, the insert was removed, rinsed by PBS, and placed in a lysis buffer to lyse the cells. The total amount of proteins in the lysate was quantitated by the BCA assay as a measurement of the cell numbers. The detected nitrite was then normalized to per cell.

\section{RESULTS AND DISCUSSION}

\section{Overview of the toolkit}

Microchannels are the key component of a microfluidic device, and they are commonly fabricated by soft lithography or 3D-printing ${ }^{1,13}$. However, these protocols demand extensive 
facilities and experienced researchers, which hinders the common adoption of microfluidic technology ${ }^{14-16}$. Nonetheless, there are readily available micro-objects in many research laboratories, such as microbore tubing, which are naturally microfluidic channels. Enlightened by this fact, we developed a toolkit that can turn microbore tubing into microfluidic devices. The core concept is that the flexible tubing can be twined, interconnected, and organized as desired via reusable adapters to form functional microfluidic units. Due to the low cost of the tubing ( 0.5 USD per $\mathrm{ft}$ ), the used segments can be disposed of while the adapters are recycled for future device assembly.

It is not practical to include all possible adapters of such a toolkit in one work. Therefore, we mainly show three subsets of the toolkit that were essential to building fundamental and functional microfluidics. The first subset included tubing and scoring tools. As demonstrated in Fig. 1A, Tygon tubing of $0.5 \mathrm{~mm}$ i.d. and $1.5 \mathrm{~mm}$ o.d. was used in this research due to availability, but tubing of other dimensions is commercially available. The ends of the tubing segments must be scored flat (rather than tapered) to minimize dead volume issues when connected to other components (adapters or tubing). Therefore, we created a scoring guider (Fig. 1Ab) with holes of $1.6 \mathrm{~mm}$ diameter (slightly larger than the tubing), where a piece of tubing could be inserted through and a sharp blade applied along the surface for a flat cut (Fig. S1 in the SI). The second subset contained various adapters for flow manipulations including joining/splitting, mixing, and adaption to detectors (Fig. 1B). We also designed a set of cell culture modules (Fig. 1C) for organs-on-a-chip applications. In the subsequent sections, design and validation details of the subsets are thoroughly discussed. Moreover, we demonstrate an application using these adapters-an integrative microfluidic device enabling 3D cell culture and near real-time quantitation.

\section{The subset of flow adapters}

This subset was designed to achieve fundamental liquid manipulations commonly used in microfluidics. There were three adapters in the subset: a T-mixer, a serpentine creator, and a Ushaped optical detector. As shown in Fig. 2A, the T-mixer had three ports to split or join flows. The flexible tubing could be inserted into the ports directly. However, for easy and leakage-free tubing integration, the dimension of the ports must be optimized. We determined the optimal diameter of the connection ports to be $1.4 \mathrm{~mm}$, slightly smaller than the diameter of the tubing $(1.5 \mathrm{~mm})$. With this size, the flexible tubing could be easily crumpled in, which then expanded to seal tightly. Port sizes between 1.3 and $1.4 \mathrm{~mm}$ could still be used but more efforts were needed 
to squeeze the tubing in. Sizes larger than $1.4 \mathrm{~mm}$ could lead to lateral leakage between the tubing and the port wall. With the tight sealing and the flat scoring of the tubing segments, the classic laminar flow could be observed (Fig. 2B) without leakage or dead volume issues at the connection sites (exemplified by arrows in Fig. 2C).

Fig. 2D shows the creator that allowed the tubing to be configured into a serpentine. Such units are usually needed on microfluidic devices to enhance liquid mixing by repeatedly shifting the flow directions ${ }^{19}$. We tested the mixing efficiency of the serpentine by pumping in two streams of food color solutions. As visualized in Fig. 2E, after five paths, the red and green streams were well mixed. Further quantitation revealed the correlation between the number of paths and the mixing efficiency (Fig. 2F).

The third flow adapter in this subset was an optical detector. As demonstrated in Fig. 3A, the adapter possessed a U-shaped groove for a piece of tubing to be fixed in. To ensure tight tubing lockage, only $1 / 4$ area along the side of the grooves $(1.5 \mathrm{~mm}$ diameter) was open (cross view in Fig. 3B), making the immobilized tubing unable to autonomously move or detach. Fig. 3C depicts the assembled device with two optical fibers placed in the side ports across the bottom part of the tubing, so that molecules of interest in the flow could be continuously monitored by absorbance detection. The dimensions of the side ports and the horizontal part of the groove were carefully designed using CAD for precise alignments. Because $1 \mathrm{~cm}$ is the commonly used light path (e.g. a standard cuvette) in absorbance spectroscopy, the horizontal part of the U-shaped groove was designed to be $1 \mathrm{~cm}$. Transparency is a key factor to be considered when developing an optical detector. Therefore, we measured the transparency of the tubing detector, finding that in the visible range $(400-800 \mathrm{~nm})$, the transmittance was higher than $85 \%$.

Instead of etching/replicating microfluidic channels in polymers using extensive facilities, these adapters in the toolkit could dexterously connect and/or configure readily available microtubing to produce desired microfluidic units. Common flow manipulators including splitter/joiner, mixer, and dynamic detectors could be robustly assembled in a plug-and-play manner. The serpentine and optical detectors were structures for twining the tubing without contacting the flowing contents. Therefore, these parts were immediately reusable. We also reused the T-mixers after bleaching and rinsing them in ultrasonication without seeing contamination. 


\section{The 3D cell culture modules}

Due to the increasing interest in integrating cells on microfluidics for organs-on-a-chip modeling ${ }^{20}$, we developed a modular chamber for 3D cell cultures in the toolkit. As shown in Fig. 4A, this chamber was assembled by three modules: a holder, an insert, and a lid. The insert was the module where cells were cultured. Following a previous protocol ${ }^{17}$, the inserts were prepared by electrospinning a layer of microfibers on a polystyrene (PS) sheet followed by being laser cut to an obround shape fitting the holder. Electrospun fibers have been extensively applied as an extracellular matrix (ECM) mimic for $3 \mathrm{D}$ cell cultures in vitro ${ }^{21}$. In this work, polycaprolactone (PCL), a commonly used biocompatible polymer 22 , was electrospun to generate a microfibrous and porous scaffold (Fig. 4B). The PS layer was used as a support structure for the flimsy microfibers to avoid flow-induced compromising.

The holder was fabricated from a flexible material with a concave obround pit $(5.0 \mathrm{~mm}$ wide, $15.0 \mathrm{~mm}$ long, $3.5 \mathrm{~mm}$ deep, Fig. 4A) in the middle, where a cell-laden insert could be placed. After the lid (3.0 mm high) was plugged in the pit of the holder, a closed chamber of 500 $\mu \mathrm{m}$ deep was generated for flow-based cell studies. Within the chamber, a flow space of $250 \mu \mathrm{m}$ was formed between the top of the insert (200 $\mu \mathrm{m}$ of PS $+50 \mu \mathrm{m}$ of the fibers; Fig. 4C) and the bottom of the lid. Simulation by computational fluid dynamics (CFD) showed that the flow rate was uniform within this flow space (Fig. 4D).

Common cell chips are fully integrated, with the cells and all other functional zones incorporated in one piece. If any part fails (e.g. cell contamination), the whole device must be discarded. The cell chamber modules we designed, however, circumvented this issue by enabling quality checking of each module before the assembly. For instance, an insert with failed cell culture could just be replaced with a new one without sacrificing the whole setup. Also, the modularity allowed for flexible integration of desired ECM materials. In our case, microfibers electrospun from PCL were utilized. Furthermore, the assembled cell chamber could be connected to other microfluidic units such as detectors via the tubing for quantitative research.

\section{Assembling a microfluidic device for near real-time cell analyses}

We validated the versatility of the toolkit by assembling a microfluidic device to quantitate the inflammatory response of macrophages. Inflammation is a critical process initiated by macrophages in response to pathogens and/or damaged tissues, and thus has become a common target for therapeutics ${ }^{23}$. Nevertheless, the detailed kinetics of the inflammatory response have remained unknown likely due to the lack of a proper analytical tool, despite this 
information being critical for finely tuning inflammations. Therefore, we applied the assembled microfluidic device to fill this knowledge gap. Inflammatory macrophages release various biomarkers, among which nitric oxide (NO) is commonly studied ${ }^{24}$. This radical molecule converts to nitrite at a $1: 1$ stoichiometry rapidly $\left(t_{1 / 2}<2 \mathrm{sec}\right)$, which can be easily assayed by the Griess reagent via the production of a pink azoic product ${ }^{25}$.

Fig. 5A outlines the workflow: phenol red-free media was pumped through the cell chamber containing macrophages; The produced nitrite was brought out to the T-mixer where the Griess reagent joined. The two solutions were further mixed via the serpentine creator to enhance the reaction and then delivered to the optical detector for near real-time measurements. Before adding cells, we first characterized the analytical merits of the device. Nitrite standards prepared in the media were pumped through, and the absorbance was detected every 20 sec. Fig. 5B clearly shows that with increased nitrite concentrations, step increased absorbance signals were obtained. After averaging the signal intensities on each plateau and plotting them vs. the nitrite concentrations, a linear calibration curve was generated with an $R^{2}$ value of 0.99 and the LOD of $0.37 \mu \mathrm{M}$. Sub- $\mu \mathrm{M}$ is the theoretical lowest LOD of UV-vis spectroscopy ${ }^{26}$. Therefore, these data suggested that our device was robust enough for the absorbance-based nitrite quantitation.

The RAW264.7 macrophage cell line, which has previously shown great success in in vitro macrophage modeling for simulating cytokine releases and inflammatory responses ${ }^{18}$, was used in this study. As shown in Fig. 5C, RAW 264.7 macrophages (arrow) could grow and fuse to form giant cells on the electrospun fibers like in vivo ${ }^{27}$. After the macrophage-laden insert was included in the device, we flowed media with or without lipopolysaccharides (LPS, an inflammation inducer ${ }^{28}$ ) through the cells, followed by automated spectroscopic detection on the detector module at intervals of $20 \mathrm{sec}$. As Fig. 5D demonstrates, the cells treated with LPS started to release significant amounts of $\mathrm{NO}$ at $30 \mathrm{~min}$ and kept secreting until a plateau of $2.8 \pm 0.1 \mathrm{nM} /$ cell at 3 hours. The unstimulated cells, however, released minimal NO during the experiments. These results were consistent with a previous study showing that macrophages in fibrous scaffolds could be stimulated to the inflammatory state by LPS within 2-4 hours ${ }^{17}$. Nonetheless, we added new knowledge here by revealing the kinetic pattern of the stimulation process in high temporal resolution (20 sec).

This cell study that included 3D cell culture, flow-based stimulation, and downstream near real-time measurements clearly demonstrated the usefulness and versatility of the toolkit to make functional and integrative microfluidic devices. Also, with the simple but robust tubing connection 
ports, we did not observe any flow issues such as leakage, blockage, and dead volumes, which allowed us to set the spectrometer and collect data without a researcher present. The simplicity and robustness of the toolkit made it possible to develop automated microfluidic analytical systems. Moreover, all the adapters and modules were reused after thorough cleaning and sterilization, and it took $<5$ min to reassemble the setup with new tubing segments, which significantly reduced both monetary and time costs as compared to current microfluidic fabrication techniques.

Although we prototyped these adapters using a high-end 3D-printer, these re-usable parts cost minimal materials ( $<5$ grams each). We have also included the engineering details of the adapters in the SI (Figs. S2-S3). With the wide availability of online 3D-printing services (user sending designs to a manufacturer and having the printed parts mailed back; also known as cloud manufacturing $)^{26}$, this technology can broadly benefit research that needs or could be enhanced by microfluidics.

\section{CONCLUSION}

We presented a new and broadly applicable toolkit for assembly of microfluidic devices without fabricating microchannels. Instead, microbore tubing, which could be twined, interconnected, and organized via the adapters for producing functional microfluidic units, was utilized as the microchannels. The low-cost tubing segments could be disposed of after usage while the adapters were reused. The robust and simple interface between the tubing and the adapters maintained consistency and interchangeability of the modules and allowed for quick assembly of devices with desired units. We demonstrated a microfluidic device assembled within 5 minutes, which enabled fluids joining/mixing, 3D cell culture, and near real-time detection. This was the first toolkit to make fully configurable microfluidic systems from microbore tubing, which featured fast and customizable prototyping, and low facility requirements. Widespread adoption of this technology will broadly enhance advances in the related fields. 


\section{FIGURES AND CAPTIONS}
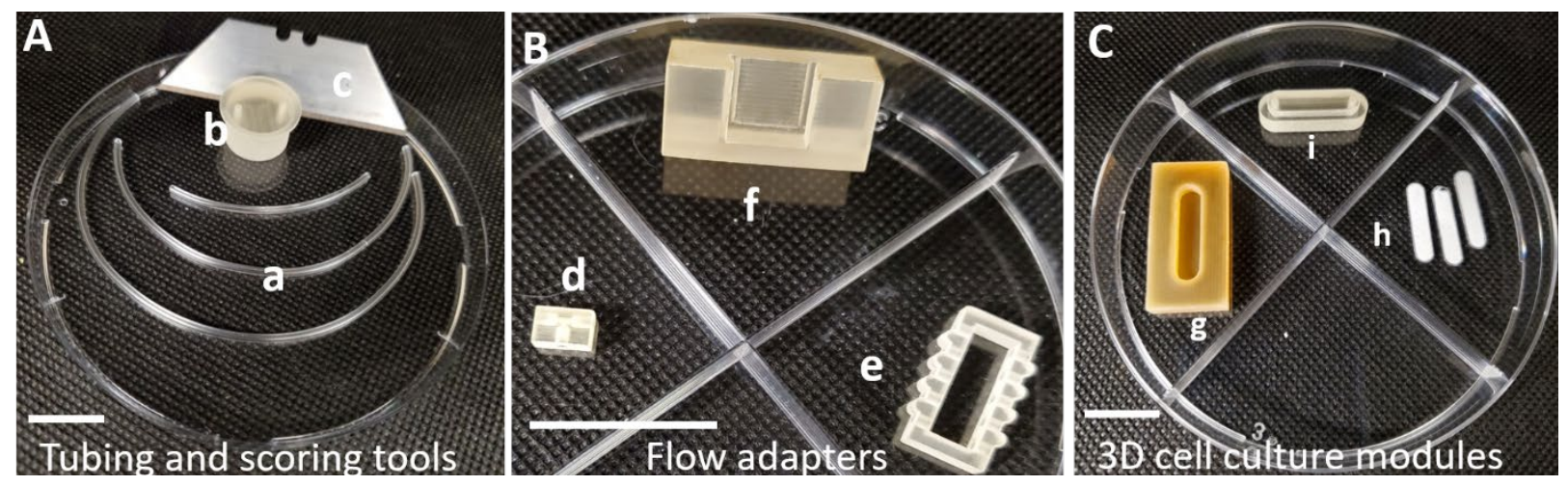

Figure 1. The three subsets of the toolkit. (A) Microbore tubing segments (a), the scoring guider (b), and a blade (c). The guider had holes of $1.6 \mathrm{~mm}$ diameter $(0.1 \mathrm{~mm}$ larger than the tubing), where a tubing segment could be inserted through. Along the surface of the guider, a blade could be utilized to score the tubing flat. (B) The flow regulators including a T-mixer with three connection ports $(d)$, a serpentine creator that could configure tubing to form a flow serpentine (e), and an optical detector that could immobilize a tubing segment in a U-shape for absorbance measurements (f). (C) The cell culture modules including a holder (g), inserts with 3D microfibrous scaffolds (h), and a lid (i). Scale bars $=1 \mathrm{~cm}$. 

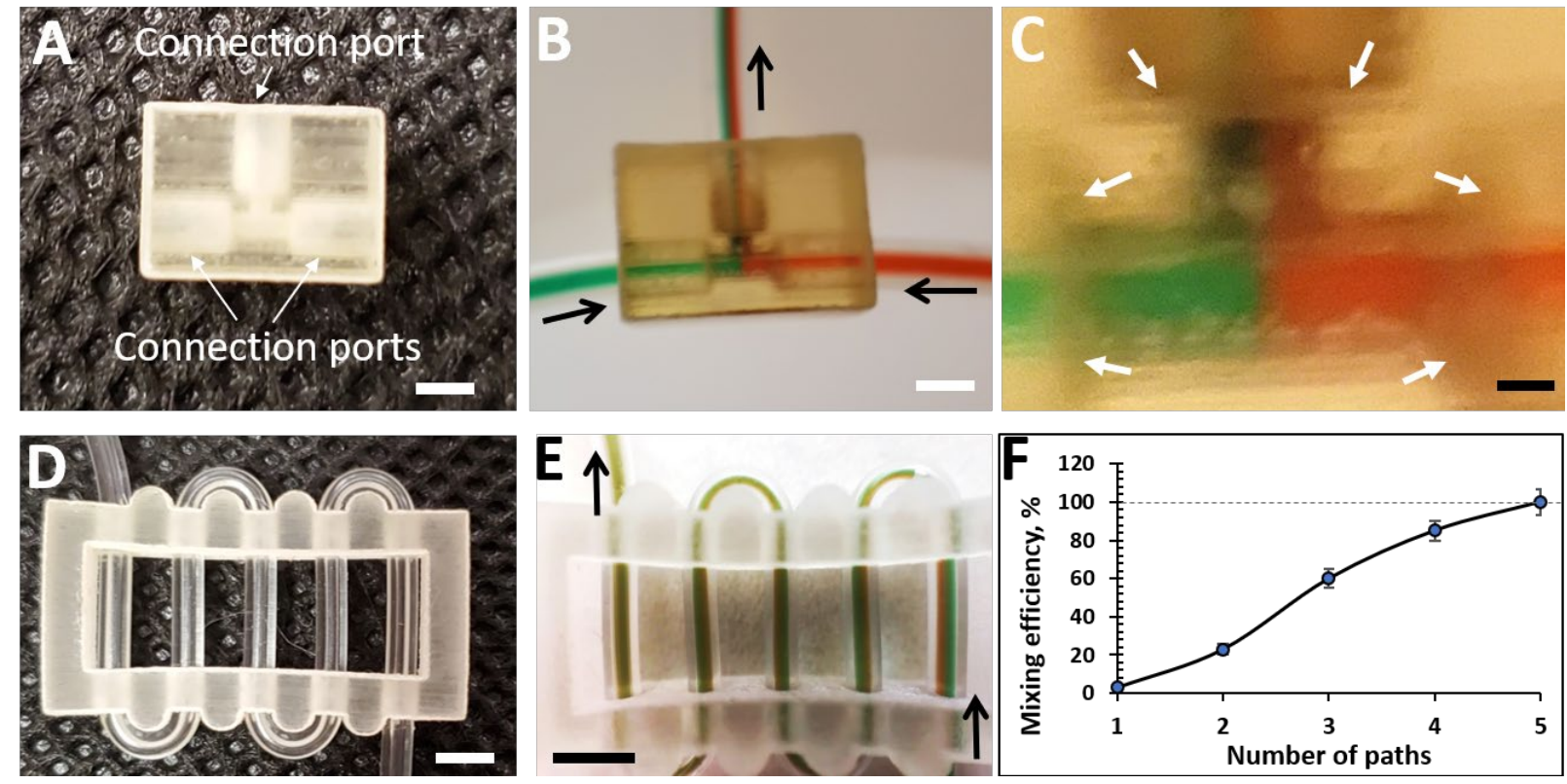

Figure 2. Details and characterization of the T-mixer and the serpentine creator. (A) The T-mixer with connection ports of $1.4 \mathrm{~mm}$ diameter, where scored tubing could be directly crumpled in. (B) In an assembled T-mixer, the classic laminar flow could be observed. Arrows show flow directions. (C) At the connection sites between the mixer and the tubing (exemplified by arrows), no leakage or dead volume issues were observed. (D) The creator that allowed the tubing to be twined to form a serpentine. (E) The laminar flows of red and green streams (the rightmost path) were completely mixed after the last (leftmost) path in the serpentine. Flow rate $=6 \mu \mathrm{L} / \mathrm{min}$. (F) The correlation between the number of paths of the serpentine and the mixing efficiency. After 5 paths, a $100 \%$ mixing was achieved; $N=5$, error $=$ stdev. Scale bars $=1 \mathrm{~mm}$. 

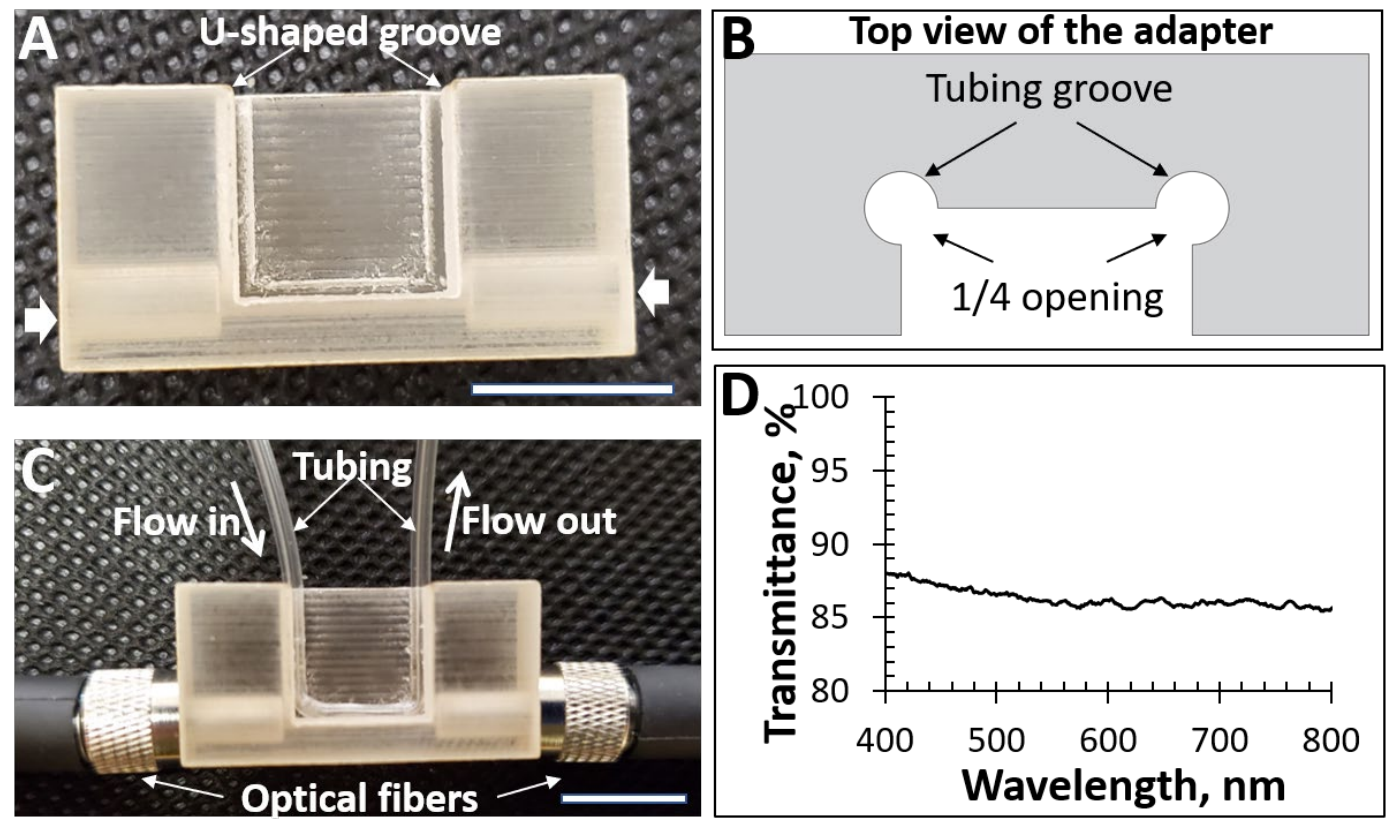

Figure 3. The adapter for optical detection. (A) The adapter with a U-shaped groove where a piece of tubing could be placed. There were two horizontal holes on the sides (arrows) to house optical fibers. Scale bar $=1 \mathrm{~cm}$. (B) The top view of the adapter shows that only $1 / 4$ of the side of the groove was open, so that the immobilized tubing would not detach or move. (C) The assembled detector. The optical fibers and the horizontal part of tubing were well aligned by carefully designing the dimensions of the housing holes and the groove. Scale bar $=1 \mathrm{~cm}$. (D) Characterization of the transparency of the detector. In the visible range, the transmittance was over $85 \%$. 

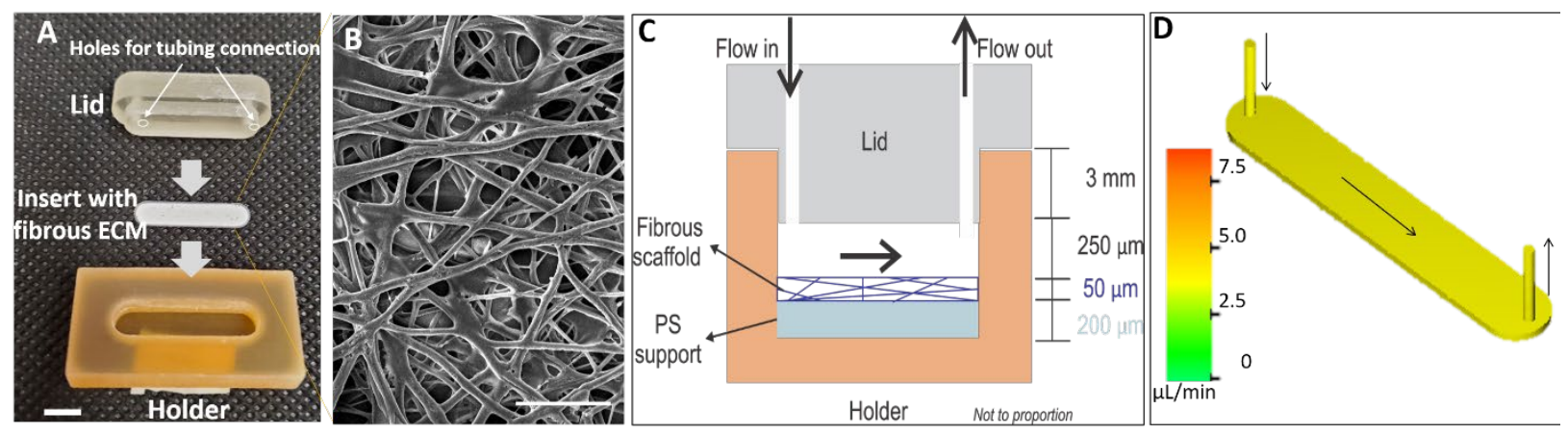

Figure 4. The 3D cell culture modules. (A) The exploded view: a holder was made from a flexible material with an obround pit, where a cell-laden insert with fibrous ECM could be placed; a lid with connected tubing was plugged in the pit to form a closed flow chamber. Scale bar $=2 \mathrm{~mm}$. (B) An SEM view of the electrospun microfibers on the insert. Scale bar $=20 \mu \mathrm{m}$. (C) An illustration of the assembled cell chamber. The pit (orange) was $3.5 \mathrm{~mm}$ deep and the lid was $3.0 \mathrm{~mm}$ high. With the inserts (200 $\mu \mathrm{m}$ PS support $+50 \mu \mathrm{m}$ fibrous scaffold), a flow space of $250 \mu \mathrm{m}$ above the fibers was formed. Arrows show the flow directions. (D) CFD simulation demonstrated that the flow rate in the chamber was uniform ( $6 \mu \mathrm{L} / \mathrm{min}$ was the simulation condition). 

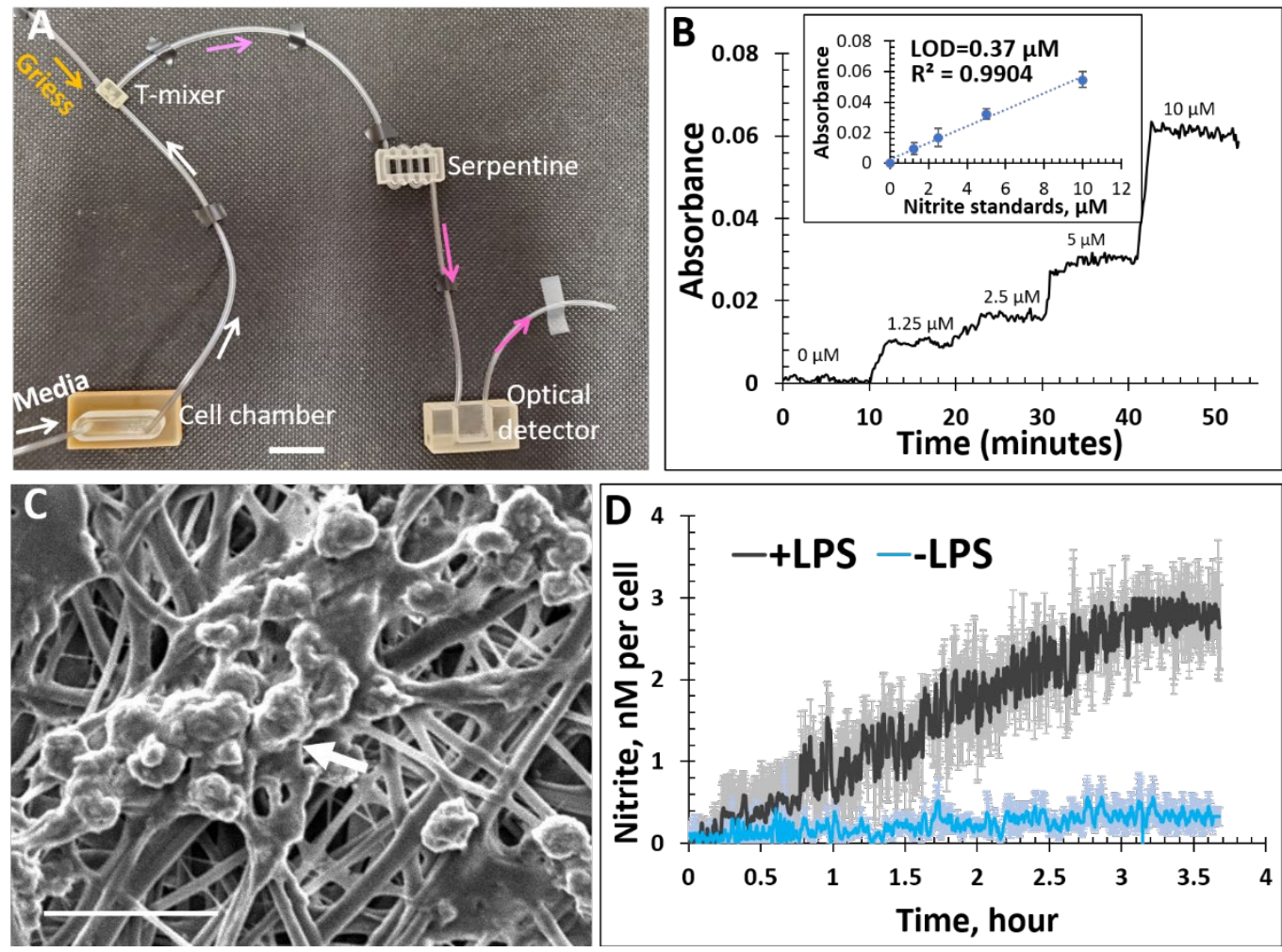

Figure 5. Using the adapters and the microbore tubing to assemble a microfluidic device for 3D cell culture and near real-time measurements. (A) The assembled device allowed media to flow through the cell chamber to the T-mixer, where the Griess reagent was mixed in. The mixture was further mixed in the serpentine and then entered the optical detector. Scale bar $=1 \mathrm{~cm}$. (B) Using nitrite standards to characterize the device (without cells). Step increased absorbance signals were obtained with increased nitrite concentrations. Plotting the average signals on each plateau as a function of the concentrations generated a linear calibration with the LOD of $0.37 \mu \mathrm{M}$. (C) Macrophages (arrow) on the fibrous ECM tended to fuse to form giant cells, similar to in vivo ${ }^{27}$. Scale bar $=20 \mu \mathrm{m}$. (D) Near real-time (20-sec intervals) quantitation of nitrite from macrophages with or without stimulation. With LPS (black line), the cells started to release significant amounts of nitrite after about 0.5 hours and continued for 3 hours until the concentration plateau appeared. For cells without the stimulation (blue line), minimal nitrite was detected. After the measurements, the cells on an insert were lysed and the total amount of proteins was detected as a gauge of cell numbers. All the nitrite data were then normalized to per cell. $\mathrm{N}=5$, error $=$ S.E.M. 


\section{REFERENCES}

[1] G.M. Whitesides, The origins and the future of microfluidics, Nature, 2006, 442(7101), 368-373.

[2] S. Lu, C.E. Dugan, R.T. Kennedy, Microfluidic Chip with Integrated Electrophoretic Immunoassay for Investigating Cell-Cell Interactions, Analytical Chemistry, 2018, 90(8), 5171-5178.

[3] B.T. Mehl, R.S. Martin, Enhanced Microchip Electrophoresis Separations Combined with Electrochemical Detection Utilizing a Capillary Embedded in Polystyrene, Analytical Methods, 2018, 10(1), 37-45.

[4] Y. Liu, X. Jiang, Why microfluidics? Merits and trends in chemical synthesis, Lab on a Chip, 2017, 17(23), 3960-3978.

[5] M. Tanyeri, S. Tay, Viable cell culture in PDMS-based microfluidic devices, Methods in Cell Biology, 2018, 148 3-33.

[6] M.H. Wu, S.B. Huang, G.B. Lee, Microfluidic cell culture systems for drug research, Lab on a Chip, 2010, 10(8), 939-956.

[7] Z.Z. Chen, W.M. Yuan, C. Xiang, D.P. Zeng, B. Liu, K.R. Qin, A microfluidic device with spatiotemporal wall shear stress and ATP signals to investigate the intracellular calcium dynamics in vascular endothelial cells, Biomechanical Modeling in Mechanobiology, 2019, 18(1), 189-202.

[8] N. Gupta, J.R. Liu, B. Patel, D.E. Solomon, B. Vaidya, V. Gupta, Microfluidics-based 3D cell culture models: Utility in novel drug discovery and delivery research, Bioeng Trans/ Med, 2016, 1(1), 63-81.

[9] M. Rothbauer, H. Zirath, P. Ertl, Recent advances in microfluidic technologies for cell-to-cell interaction studies, Lab on a Chip, 2018, 18(2), 249-270.

[10] J.C. McDonald, D.C. Duffy, J.R. Anderson, D.T. Chiu, H. Wu, O.J. Schueller, G.M. Whitesides, Fabrication of microfluidic systems in poly(dimethylsiloxane), Electrophoresis, 2000, 21(1), 27-40. [11] B.C. Gross, J.L. Erkal, S.Y. Lockwood, C. Chen, D.M. Spence, Evaluation of 3D printing and its potential impact on biotechnology and the chemical sciences, Analytical Chemistry, 2014, 86(7), 3240-

53.

[12] A.K. Au, W. Huynh, L.F. Horowitz, A. Folch, 3D-Printed Microfluidics, Angewandte ChemieInternational Edition, 2016, 55(12), 3862-3881.

[13] C.P. Chen, B.T. Mehl, A.S. Munshi, A.D. Townsend, D.M. Spence, R.S. Martin, 3D-printed microfluidic devices: fabrication, advantages and limitations-a mini review, Analytical Methods, 2016, 8(31), 60056012.

[14] C.E. Owens, A.J. Hart, High-precision modular microfluidics by micromilling of interlocking injectionmolded blocks, Lab on a Chip, 2018, 18(6), 890-901.

[15] K. Vittayarukskul, A.P. Lee, A truly Lego-like modular microfluidics platform, Journal of Micromechanics and Microengineering, 2017, 27(3), 035004.

[16] A.S. Munshi, C.P. Chen, A.D. Townsend, R.S. Martin, Use of 3D printing and modular microfluidics to integrate cell culture, injections and electrochemical analysis, Analytical Methods, 2018, 10(27), 33643374.

[17] C.P. Chen, A.D. Townsend, E.A. Hayter, H.M. Birk, S.A. Sell, R.S. Martin, Insert-based microfluidics for 3D cell culture with analysis, Analytical and Bioanalytical Chemistry, 2018, 410(12), 3025-3035.

[18] B. Taciak, M. Bialasek, A. Braniewska, Z. Sas, P. Sawicka, L. Kiraga, T. Rygiel, M. Krol, Evaluation of phenotypic and functional stability of RAW 264.7 cell line through serial passages, PLoS One, 2018, 13(6), e0198943.

[19] Y. Huang, Y. Wang, L. Xiao, H. Liu, W. Dong, Z. Yin, Microfluidic serpentine antennas with designed mechanical tunability, Lab on a Chip, 2014, 14(21), 4205-12.

[20] S. Ishida, Organs-on-a-chip: Current applications and consideration points for in vitro ADME-Tox studies, Drug Metabolism and Pharmacokinetics, 2018, 33(1), 49-54. 
[21] I.J.H. Barrientos, G.R. MacKenzie, C.G. Wilson, D.A. Lamprou, P. Coats, Biological Performance of Electrospun Polymer Fibres, Materials, 2019, 12(3).

[22] J.R. Venugopal, Y. Zhang, S. Ramakrishna, In vitro culture of human dermal fibroblasts on electrospun polycaprolactone collagen nanofibrous membrane, Artificial Organs, 2006, 30(6), 440-6. [23] T.A. Wynn, L. Barron, Macrophages: master regulators of inflammation and fibrosis, Seminars in Liver Disease, 2010, 30(3), 245-57.

[24] J. MacMicking, Q.W. Xie, C. Nathan, Nitric oxide and macrophage function, Annual Review of Immunology, 1997, 15 323-50.

[25] D. Tsikas, Analysis of nitrite and nitrate in biological fluids by assays based on the Griess reaction: appraisal of the Griess reaction in the L-arginine/nitric oxide area of research, J Chromatogr B, 2007, 851(1-2), 51-70.

[26] W. Mantele, E. Deniz, UV-VIS absorption spectroscopy: Lambert-Beer reloaded, Spectrochimica Acta Part A: Molecular and Biomolecular Spectroscopy, 2017, 173 965-968.

[27] A. Vignery, Macrophage fusion: the making of osteoclasts and giant cells, The Journal of Experimental Medicine, 2005, 202(3), 337-40.

[28] C. Chen, B.T. Mehl, S.A. Sell, R.S. Martin, Use of electrospinning and dynamic air focusing to create three-dimensional cell culture scaffolds in microfluidic devices, Analyst, 2016, 141(18), 5311-20. 